\title{
Non-Traumatic Lateral Hip Pain: MRI Findings
}

\author{
SHERIF A. ABO-HEDIBAH, M.D.*; WALAA A. GOUDA, M.D.** and SAMEH A. ALY, M.D.*** \\ The Department of Radiodiagnosis, Faculty of Medicine, Cairo*, Menofia** and Banha*** Universities
}

\begin{abstract}
Background: Imaging findings play important role in diagnosis and directing the treatment for lateral hip pain. MRI plays a crucial role in the structural diagnoses accompanyinglateral hip pain include tendinosis, and partial- or fullthickness tears of the gluteal tendons.

Aim of Study: Todescribe the MRI findings related to the non-traumatic lateral hip pain.

Patients and Methods: A retrospective study was performed including forty patients ( 35 females and 5 males, between the ages of 23 and 76 the mean \pm SD, 50.1 \pm 13.6 years) presented with unilateral or bilateral LHP and all patients underwent MRI for both hips. Two radiologists reviewed the MR images for identification of the lateral hip pathology.

Results: In the 40 patients included in our study, there were (16) patients complaining of unilateral hip pain and (24) patients complaining of bilateral hip pain. (34) patients showed MRI features of tendon, muscle \& bursal pathologies (either unilateral or bilateral), (4) patients showed AVN of the painful hip and (2) cases were diagnosed as transient migratory osteoporosis.

Conclusion: MRI is a valuable method to identify the structural diagnoses accompanying lateral hip pain.
\end{abstract}

Key Words: MRI-Lateral hip pain.

\section{Introduction}

LATERAL hip pain (LHP), which is commonly referred as "greater trochanteric pain syndrome," is frequently encountered by physical physician. It can be exemplified by episodes of pain in the vicinity of the greater trochanter. This condition has also been labeled a regional pain syndrome by some due to its nonspecific cause [1-3]. Diagnosis of LHP etiology is challenging, because the pain may be referred to this area from the lumbar region, or may arise secondary to abnormalities of numerous intra-articular or periarticular structures [2-4]

Correspondence to: Dr. Sherif A. Abo-Hedibah, The Department of Radiodiagnosis, Faculty of Medicine, Cairo University
Diagnosis is typically based on the patient history and physical examination, with trochanteric bursitis previously being considered as the primary cause of symptoms [5-7]. However, recent imaging studies [8,9] have raised questions about which structures are implicated in this condition, with interest shifting towards the role which the gluteal tendons may truly have in causing LHP [10-12]

Few studies have evaluated gluteal tendons pathology as a reason of LHP, yet the etiology and pathogenesis of this condition have to be determined. In patients with LHP the prevalence of gluteal tendon pathology is variable, ranging between $27.6 \%$ and $84.2 \%$ [13-16].

Our study aimed to evaluate the role of MRI in diagnosis of non-traumatic lateral hip pain after exclusion of referred pain (i.e. pain from the lumbar spine).

\section{Patients and Methods}

\section{Patients:}

This retrospective study was conducted in the period between January 2017 and June 2018 includingforty patients [ 35 females and 5 males, between the ages of 23 and 76 (mean \pm SD, $50.1 \pm 13.6$ years)] who presented with LHP and referred from the orthopedic outpatient clinic in Farwaniya Hospital/Kuwait for MRI evaluation of both hips.

The study was approved by our institutional review boardand all patients were given a written informed consent before being included in the study and all their rights were protected.

Patientsexcluded from our study:

- Patients under 18 years old.

- Patients gavehistory of trauma to the hip joint. 
- Who received a steroid injection in the region of the greater trochanter in the preceding 6 months.

- Patient undergone previous hip joint or pelvic surgery.

- Had lumbar pathology with referred LHP.

- Or/if MRI was contraindicated, if the patient had: Cardiac pacemaker, history of intracranial vessels clipping, any other small metal implants or wires that could be affected by MR field. As well as claustrophobic and pregnant patients.

\section{MRI technique:}

MRI images were acquired on a 1.5-T Signa Infinity whole body system with Excite, Version 11 (General Electrical Medical Systems, Milwaukee, WI). Axial and coronal images of the pelvis and both hips were taken simultaneously, with the patient lying supine. A torso phased array, receiveonly coil, was used to encode the signal.

The scanning level startsfrom the level of anterior superior iliac spine till the upper shaft of the femur, and from the anterior superior iliac spine to the ventral surface of the sacrum posteriorly. The time taken for the imaging studies was between averaged 30-40 minutes per patient.

\section{Parameters:}

1- Fast spin echo axial and coronal T1-weighted images (repetition time, 600 milliseconds; echo time, 9.9 milliseconds; echo train length,2;5$\mathrm{mm}$ slice thickness with 1-mm inter-slice gap; $36-\mathrm{cm}$ field of view; 352 x 256 matrix).

2- Axial and coronal T2-weighted fast spin-echo fat-saturated (repetition time, 3000 milliseconds; echo time, 15-20 milliseconds; echo train, 15; 5-mm slice thickness, 1-mm inter-slice gap; 36$\mathrm{cm}$ field of view; $256 \times 256$ matrix).

3- Coronal and axial proton density fat-suppressed images (PD Fat SAT) (repetitiontime, 3925 milliseconds; echo time, 85 milliseconds; echo train, $15 ; 5-\mathrm{mm}$ slice thickness, 1-mm interslice gap; 36-cm field of view; 288 x 256 matrix)

\section{Data interpretation:}

The images of the right and left hips from each participant were analyzed by 2 consultant radiologists with 13 and 19 years of experience, respectively. The MRI images were viewed for the presence of any hip pathology including gluteal tendon, bursae, osteoarthritic changes, and any other pathology observed in the region of the hip.

\section{Anatomical consideration:}

Although variable bursae are seen related to the lateral aspect of the hip, the commonly encountered bursae are three: Greater trochanteric, subgluteus medius, and subgluteus minimus bursae (Fig. 1) [4].

The gluteus minimus and medius tendons forming the "rotator cuff of the hip" and are inserting onto the greater trochanter. The surface of the greater trochanter contains four discrete facets: Anterior, posterior, lateral, and superoposterior. The anterior facet carries the insertion of the gluteus minimus while the gluteus medius attaches to the superoposterior and lateral facets (Figs. 2,3) [4]

Tendinos is was diagnosed whenthere is increased thickness and signal intensity of the tendon on PD Fat Sat images. The presence of fluid signals interrupting the fibers represented a partial tendon tear. A complete tendon tear was recorded if the distal tendon was completely disrupted on T1weighted images, accompanied by a marked increased signal on PD Fat Sat images which may be associated with atrophy of the muscle.

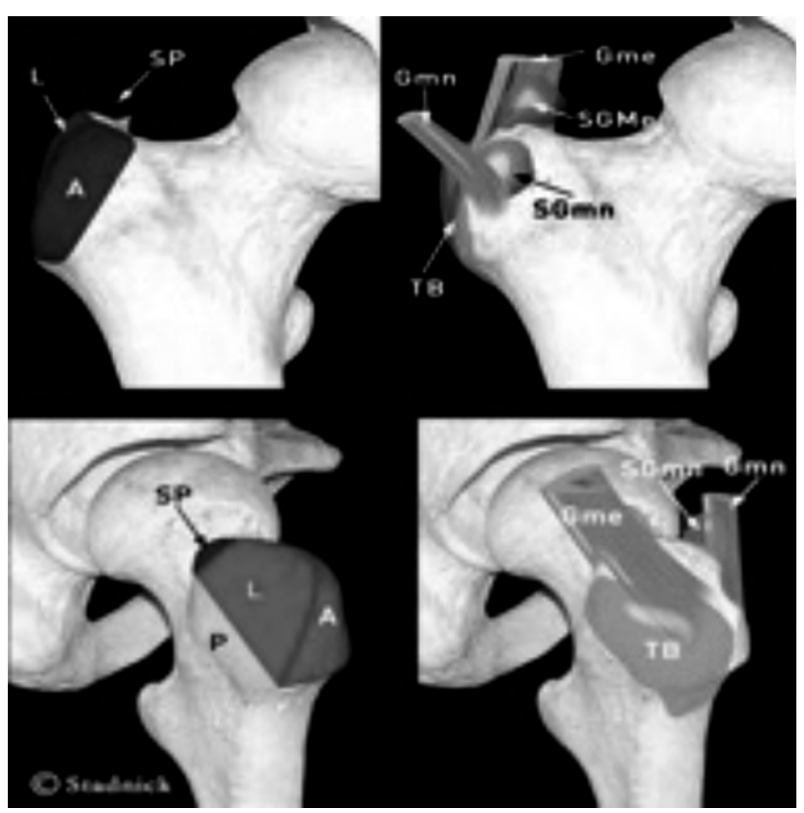

Fig. (1):Frontal and lateral graphsdemonstrate the anatomy at the greater trochanter facets: Anterior (A), lateral (L), posterior $(\mathrm{P})$, and superoposterior (SP).The trochanteric (TB), subgluteus minimus (SGmn), and subgluteus medius (SGMe) bursae are labelled by blue. Gluteus minimus (Gmn) and gluteus medius (Gme) tendons [4]. 

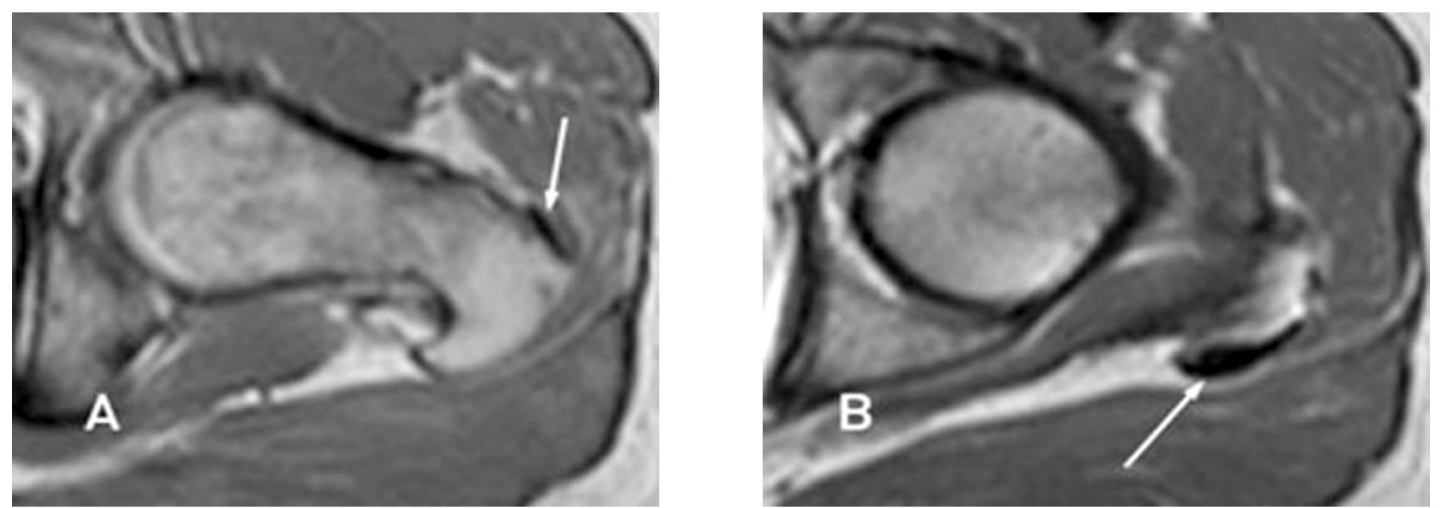

Fig. (2): T1-weighted axial images shows the normal appearance of: (A) The insertion of the gluteus minimus tendon (arrow) onto the greeter trochanter anterior facet. (B) The gluteus medius tendon insertion (arrow) onto the greater trochanter superoposterior facetat a higher level [4]

(A)

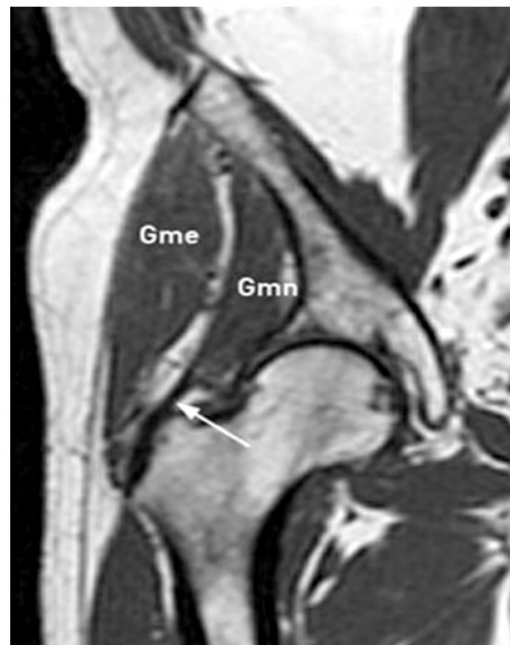

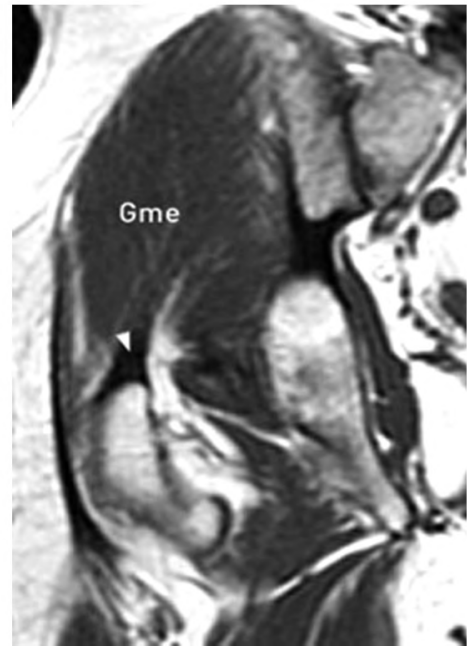

(B)

Fig. (3): T1-weighted coronal images show: (A) The gluteus minimus tendon Gmn (arrow) inserting on the anterior portion (top) of the facet of the greater trochanter. (B) The gluteus medius tendon Gme (arrowhead) inserting on the greater trochanter posterior portion (bottom) [4]

Bursitis was diagnosed if a bursa appeared distended and displayed isointensity relative to the contents of the urinary bladder on T2-weighted and PD Fat Sat images with hypointensity on T1weighted images. The specific anatomical location of the pathological bursa (e) was verified when MRI findings of bursitis was apparent. If the region of altered signal intensity was lateral to the surface of the greater trochanter, underneath the fascia lata and gluteus maximus, yet superficial to the distal tendon of gluteus medius the bursa (e) involved was reported as the "trochanteric bursa". Altered signal in the bursae at the superior border or apex of the greater trochanter and lying deep to the tendons of gluteus mediusand gluteus minimus at their trochanteric insertion were reported as the "subgluteus medius" and "subgluteus minimus" bursae, respectively. Joint space narrowing, osteophytes, subchondral sclerosis, and subarticular cysts denoting hip osteoarthritis. Any other abnormalities that presented in the region of the hip joint, including avascular necrosis, iliopsoas bursa enlargement, muscle atrophy and joint effusion were also reported.

\section{Results}

The (40) patients included in our study showed variable unilateral or bilateral lateral hip joint pain. There were (16) patients complaining of unilateral hip pain and (24) patients complaining of bilateral hip pains.

(34) patients show MRI features of tendon, muscle \& bursal pathologies (either unilateral or bilateral), (4) patients show AVN of the painful hip and (2) cases of transient migratory osteoporosis.

So, the tendon, muscle $\&$ bursal pathologies represent $85 \%$ of the cases of LHP and the AVN represents $10 \%$, while transient migratory osteoporosis represents $5 \%$ of cases (Fig. 4). 
When we analyzed the tendon, muscle and bursal pathology, we found that 23 cases $(68 \%)$ show tendon pathology (i.e. 15 cases of gluteus medius and minimus tendinosis, 4 cases with partial gluteus minimum tear, 2 cases with gluteus

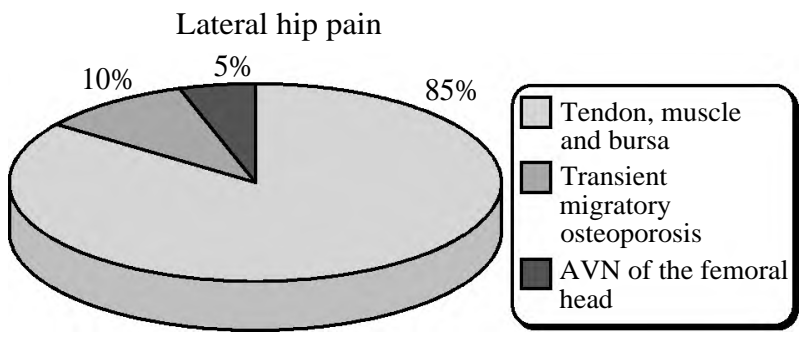

Fig. (4): A pie chart showing the percentage of the different causes of LHP in our study.
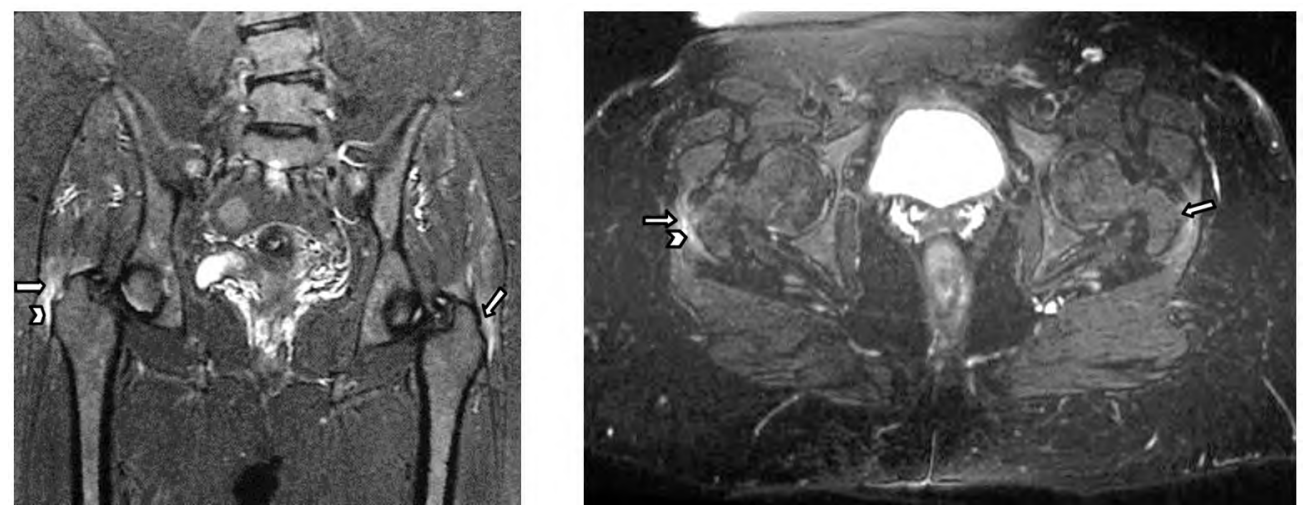

Fig. (6): 59- year-old female patient presented with chronic bilateral lateral hip pain. Coronal and axial PD Fat Sat images show bilateral abnormal high signal intensity in both gluteus medius tendons (white arrows), with the right one shows linear fluid signal interrupting some of its fibers near the insertion denoting partial tear (arrow head).
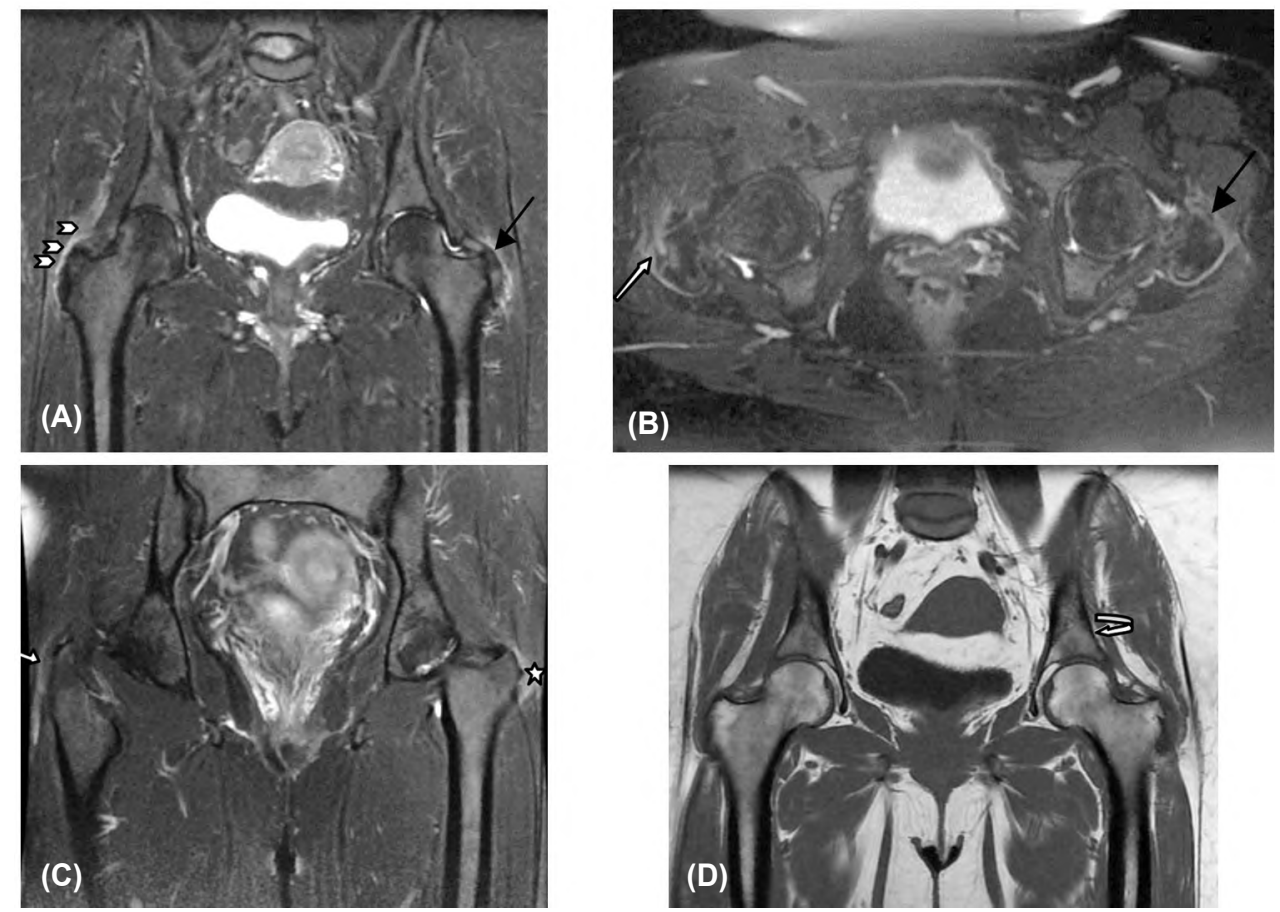

Fig. (7): 54- year-old female patient presented with chronic bilateral lateral hip pain. (A\&C) coronal and (B) axial PD Fat Sat images show:increased thickness of the left gluteus minimus tendon with abnormal high signal intensity denoting tendinosis (black arrow) and minimal surrounding fluid (peritendinitis). Also noted increased signal of the right gluteus medius muscle (tendinosis, white arrows) with mild fluid below(mildright subgluteus medius bursitis, arrow heads) and mild left greater trochanteric bursitis (star). (D) coronal T 1 image showed diffuse atrophy of the left gluteus minimus tendon (curved arrow). 

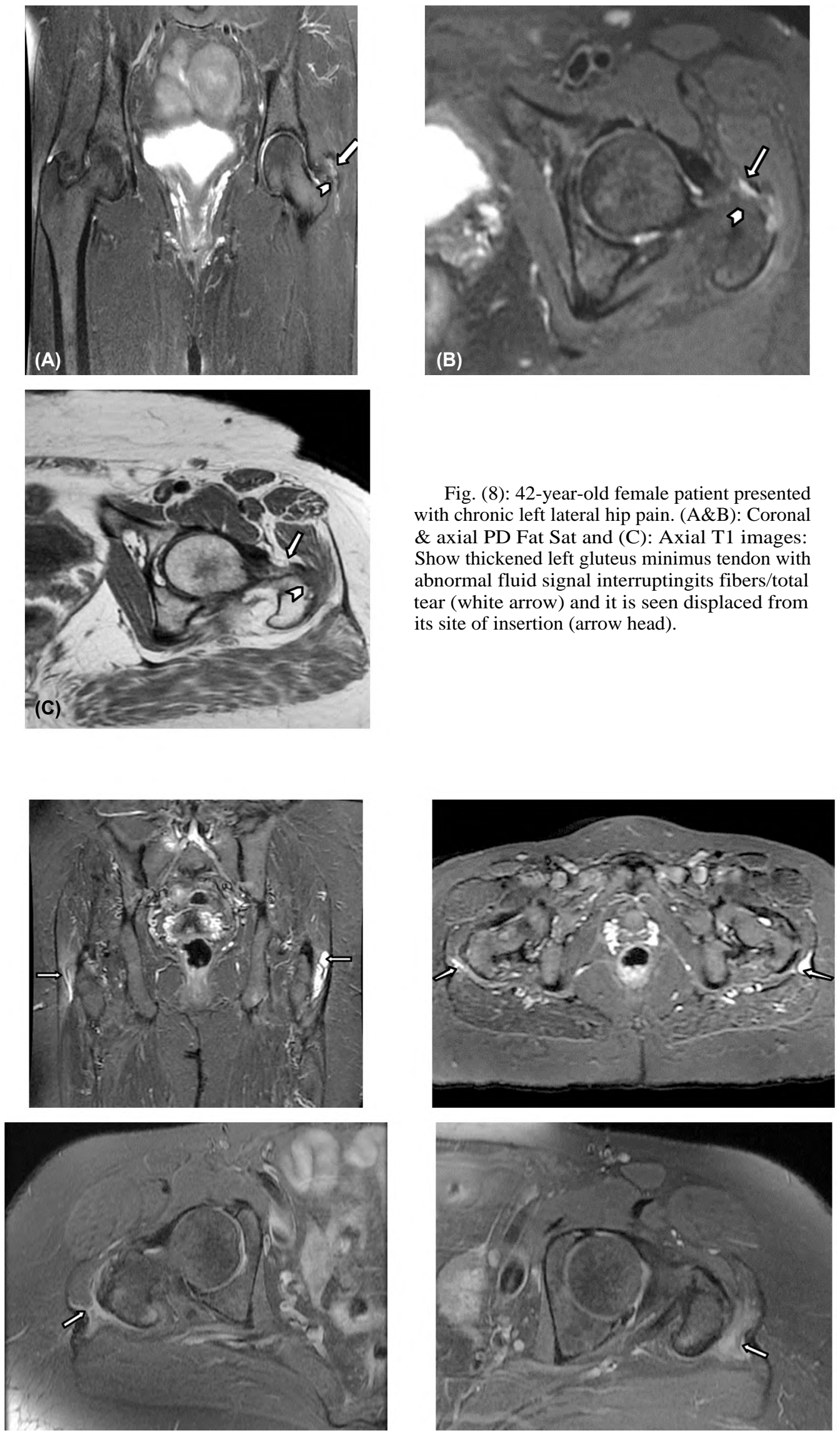

Fig. (8): 42-year-old female patient presented with chronic left lateral hip pain. (A\&B): Coronal $\&$ axial PD Fat Sat and (C): Axial T1 images: Show thickened left gluteus minimus tendon with abnormal fluid signal interruptingits fibers/total tear (white arrow) and it is seen displaced from its site of insertion (arrow head).

Fig. (9): MRI of both hips (Coronal \& axial PD Fat Sat images) showing bilateral greater trochanteric bursitis more on the left side (white arrows). 

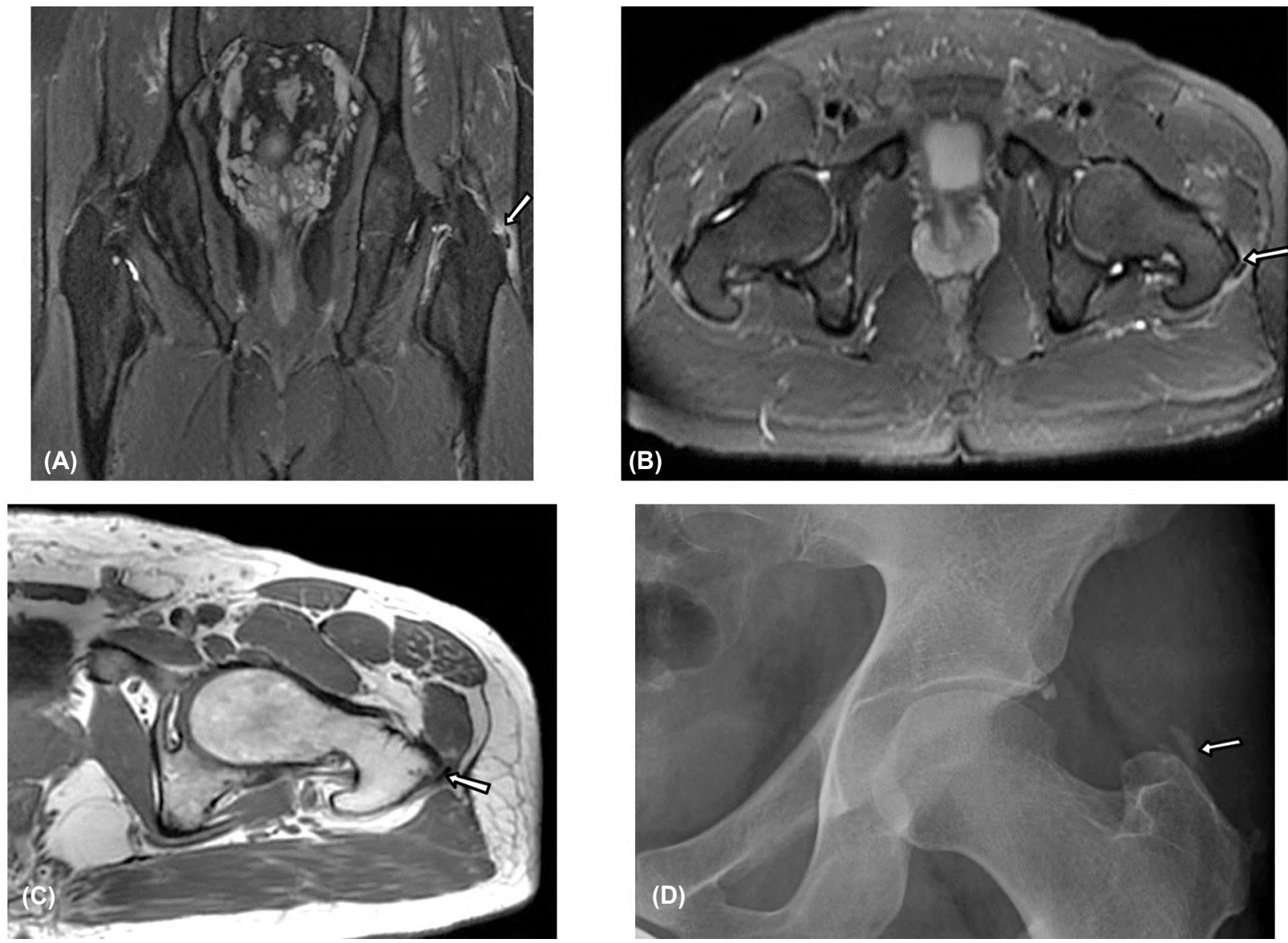

Fig. (10): 49-year-old male patient presented with chronic left lateral hip pain. A case of left gluteus medius calcific tendinitis (A) Coronal \& (B) axial PD Fat Sat imagesshowing abnormal high signal intensity within the left gluteus medius tendon with low signal intensity elongated focus (arrow) and minimal surrounding fluid (peritendinitis). (C) axial T1 WI of the left hip showing the low signal intensity focus. (D) X-ray/AP view of the left hip showing elongated calcific focus at the superior portion of the greater trochanter (arrow).
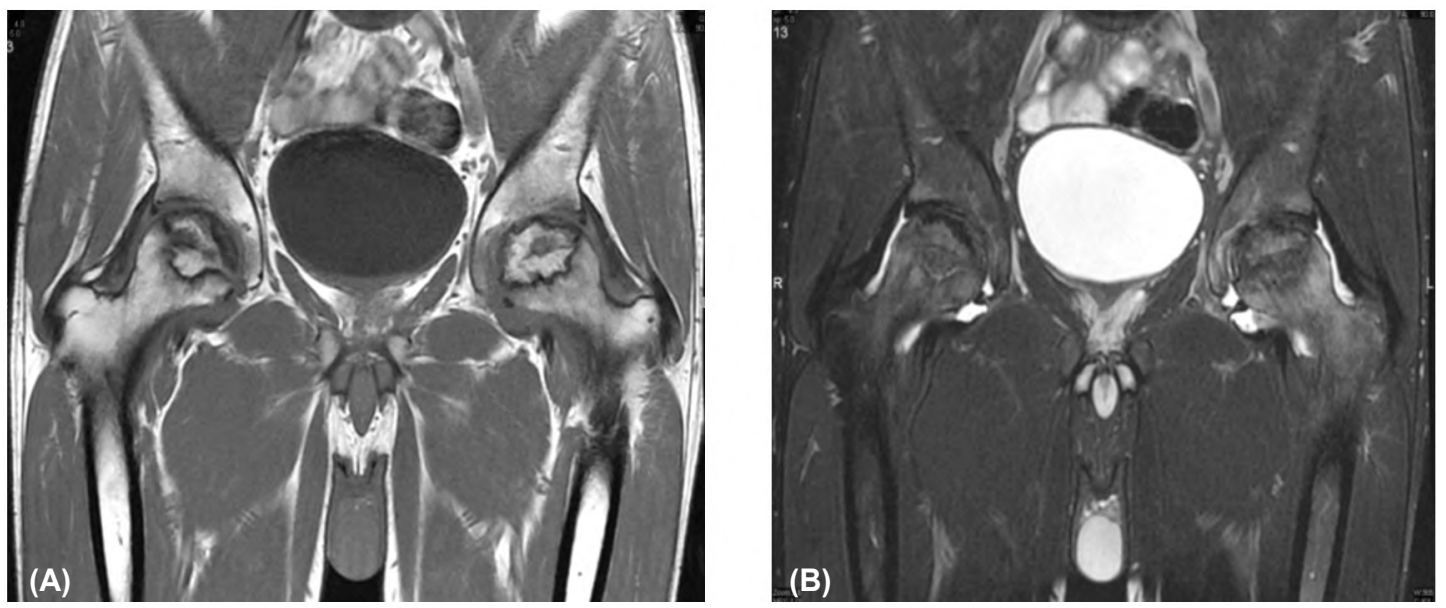

Fig. (11): 44-year-old male patient presented with chronic bilateral lateral hip pain (a case of AVN of bilateral femoral heads). Coronal T1 WI (A) \& coronal PD-FS WI (B): Showing abnormal bone marrow signal of both femoral heads surrounded by curved zone of low T1 and high Pd-FS signal intensity and associated with minimal bilateral hip joint effusions. 

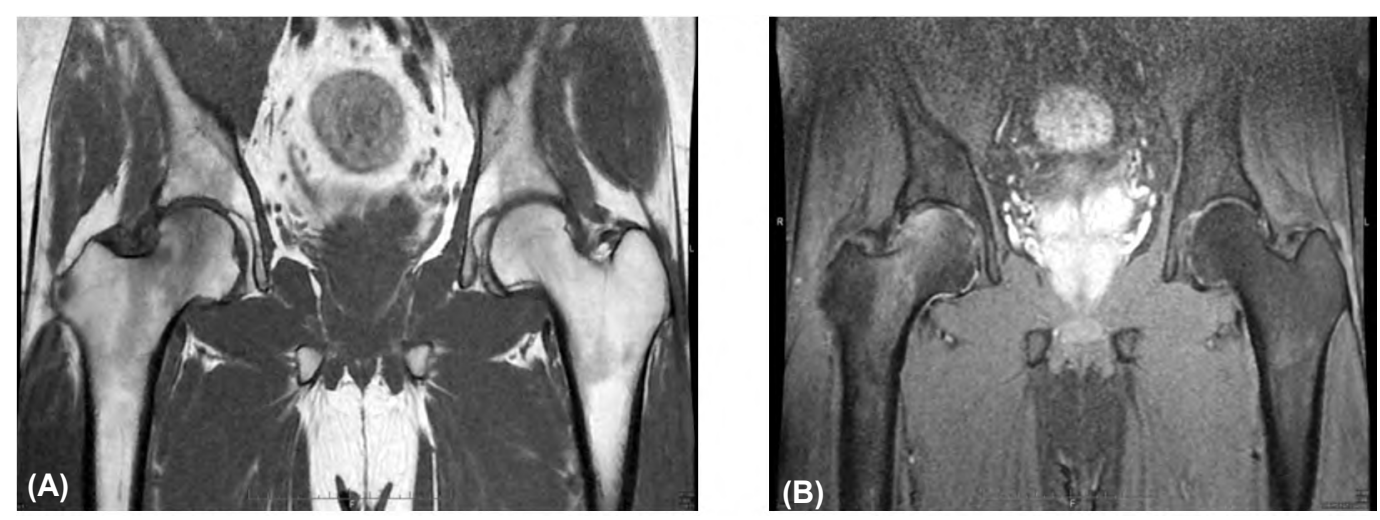

Fig. (12): 37-year-old male patient presented with chronic right lateral hip pain (acase of transient migratory osteoporosis of the right femoral head). Coronal T1 WI (A) \& coronal PD-FS WI (B) showing abnormal bone marrow signal of the right femoral head of low T1 and high Pd-FS signal intensity with no definite transitional zone separating it from the rest of the normal femoral bone marrow signal intensity.

\section{Discussion}

Lateral hip pain affecting about $10 \%$ to $25 \%$ of the general population. Pain alongthe greater trochantergenerally referred as greater trochanteric pain syndrome. At the lateral hip region, many pathologies can be involved in pain pathogenesis, including related bursitis, tendinosis of the gluteus medius and minimus muscle tendonsand iliotibial band thickening. Patients may complainfrom mildmorning stiffness andmight be unable to sleep on the affected side. Gluteus minimus and medius tendon pathology commonly manifest by pain along the anterior and posterolateral aspect of the lateral hip. Most of the patients show no history of trauma and their symptoms have insidious onset owing to the repetitive use [9].

So lateral hip pain involves not only trochanteric bursitis but also what have been labelled aship rotator cuff tears that refers to a tear in one or more tendons of the hip abductors and analogous to shoulder rotator cuff tears $[3,12]$.

The trochanteric bursal complex encompasses the major bursae around the hip including; subgluteus medius and maximus bursae and a solitary minor bursa: Subgluteus minimus. Studies investigative the trochanteric bursal complex revealed that tendinopathy of the gluteus medius and minimus muscles in these patients is a part of a spectrum of injury, starting with bursitis complicating impingement, followed by tendinopathy and the formation of tears [11-15].

Bursitis related to the lateral aspect of the hip is diagnosed by distension of the trochanteric, subgluteus maximus or medius bursaeby fluid signal on MR weighted images. This finding is quite commonly absent in pateints with lateral hip pain, that is why greater trochanteric pain syndrome is now widely used rather than trochanteric bursitis. Up to $40 \%$ of patient's bursitis has also been found to co-exist with gluteal tendon pathology [3]

Tendinopathy is a degenerative condition commonly affecting athletes because of chronic overuse of a given tendon or musculo-tendinous unit. The onset of the symptoms is usually insidious including pain, which is aggravated by resisted function. In general, tendinopathy on MRI exhibits focal or diffuse thickening of the tendon with increased PD Fat Satsignal. Small, recurrent intra-substance collagen fibril tears may contribute to chronic tendinopathy, occasionally seen on imaging as focal linear intra-substance fluid signal without acute injury. Partial thickness tears demonstrate focal discontinuity and high T2 signal, and fullthickness tears are easily identified as a wavy hypointense tendon, fluid-filled gap at the tear site, and possible tendon retraction [10].

Gluteal tears typically manifested as a defect most commonly in the gluteus minimus tendon \& commonly involving the lateral part of the gluteus medius tendon. A partial tear is diagnosed where focal tendon disruption is present and complete tear when full thickness abnormality or tendon retraction is depicted. Bald trochanter is a term used when there is full thickness tears of both gluteus medius and minimus with tendon retraction obvious on MR. Partial tears can be isolated but usually occur on a background of underlying gluteal tendinosis or tendinopathy [14].

Diagnostic images play crucial role in the differentiation of multiple hip pathologies with the Magnetic Resonance Imaging (MRI), is considered one of the main diagnostic tools for hip joint painassessment. It offers detailed information 
regarding the different structures including the articulating bones, muscles and tendons as well as, nerves and surrounding soft tissues [6].

In our study, we found that atraumatic lateral hip is mostly attributed to tendon, bursal or muscle pathology (85\%), with the tendon pathology were the commonest among them (68\% of patients).

MR imaging was found to be the modality of choice for evaluation of abductor tendons pathology through the documented previous study, with a $95 \%$ specificity and $91 \%$ sensitivity for the diagnosis of tendons pathology [9]

In this study we reported the imaging findings of tendon pathology based on the identification of tendinosis, partial and full-thickness tears, separately. We reported 15 cases of gluteus medius and minimus tendinosis, 4 cases with partial gluteus minimus tear, 2 cases with gluteus maximus partial tear, 1 case gluteus minimus tendon total tear and 1 case with gluteus medius calcific tendinitis.

We found that for correctdiagnosis of the abductor tendons pathology, the radiologist has to know and assess carefully the abductor tendons and their site of attachment.

The attachment of the gluteus minimus tendonis seen at the greater trochanter anterior facet and at the capsule of the hip joint. The major attachment of the gluteus medius tendon is seen at the greater trochanter superoposteriorandlateral facet. A trochanteric bald areais found near the top of the greater trochanter lateral facet, which is an area lacking tendon insertion. This bald areais about $21 \mathrm{~mm}$ in average diameter, covered by the subgluteus medius bursa, bordered by the gluteus minimus, the gluteus medius, and the piriformis tendon and that should not be mistaken for gluteal tendon tear. Peri-trochanteric soft-tissue abnormalities with mild increased T2 signal intensity in MR images are commonly found in $20 \%-88 \%$ of asymptomatic patients. Also reported that in one-third of patients who undergo contrast-enhanced pelvic MR imaging for non-musculoskeletal conditions, there is mild diffuse peri-trochanteric post contrast enhancement between the gluteus medius tendon and the iliotibial band. These abnormalities should not be mistaken for trochanteric bursitis [11].

So MRI allows accurate detection of tears of the abductor tendons and allows effective treatment planning in such cases.

Steinert et al., 2010 [7], showed similar results and revealed that tendinopathy of the hip abductor tendons is becoming increasingly documented as a common cause of lateral hip pain. The trochanteric bursitis was frequently found as manifestation of underlying abductor tendon pathology rather than, being the primary abnormality. The presence of trochanteric bursitis should thus prompt a careful assessment of the abductor tendons.

\section{Conclusion:}

MRI show a good ability to identify different pathology contributing lateral hip with the gluteal tendon pathology was the commonest MRI findings in our patients.

\section{Conflict of interest:}

Non declared.

\section{References}

1- DRAR H., MOHAMMED B., ALI Z., et al.: The role of MRI in the evaluation of painful hip joint (MRI of hip joint). International Journal of Medical Imaging, 2 (3): 77-82, 2014

2- LADD L.M, BLANKENBAKER D.G., DAVIS K.W., et al.: MRI of the Hip: Important Injuries of the Adult Athlete. Current Radiology Reports, 2 (51): 1-19, 2014.

3- MacMAHON P.J., HODNETT P.A., KOULOURIS G.C., et al.: Hip and Groin Pain: Radiological Assessment. The Open Sports Medicine Journal, 4: 108-120, 2010.

4- MANASTER B.J.: Adult Chronic Hip Pain: Radiographic Evaluation. Radio Graphics, 20: S3-S25, 2000.

5- MULLIGAN E.P., MIDDLETON E. and BUNDY B.: Greater Trochanteric Pain Syndrome: Antalgic Hips in an Active Population with Athletic Aspirations. American Physical therapy Association (APTA) Combined Sections Meeting, Indianapolis, 7, 2015.

6- RUMIE C., VASQUEZ A., ABREU J.A., et al.: Magnetic Resonance in Hip Pain Assessment: Beyond the Femoroacetabular Impingement. European Society of Radiology, 10: 1594-2118, 2015.

7- STEINERT L., ZANETTI M., HODLER J., et al.: Are radiographic trochanteric surface irregularities associated with abductor tendon abnormalities? Radiology, 257 (3): 754-763, 2010.

8- SUTTER R., ZANETTI M. and PFIRRMANN C.W.: New Developments in Hip Imaging. Radiology, 264 (3), 2012.

9- WILSON J.J. and FURUKAWA M.: Evaluation of the Patient with Hip Pain. American Family Physician web site, (89): 1, 2014.

10- WOODLEY S.J., NICHOLSON H.D., LIVINGSTONE V., et al.: Lateral Hip Pain: Findings from Magnetic Resonance Imaging \& Clinical Examination. Journal of orthopaedic \& sports physical therapym 38 (6): 313-328, 2008.

11- PFIRRMANN C.W., CHUNG C.B., THEUMANN N.H., et al.: Greatertrochanter ofthehip: Attachmentoftheabductormechanismandacomplexof three bursae-MR imaging and MR bursography in cadavers and MR imaging in asymptomatic volunteers. Radiology, 221 (2): 469-77, 2001. 
12- CVITANIC O., HENZIE G., SKEZAS N., et al.: MRIdiagnosisoftearsofthe hip abductor tendons (gluteus medius and gluteus minimus). AJR. Am. J. Roentgenol., 182 (1): 137-43, 2004.

13- MOOSMAYER S., TARIQ R., STIRIS M., et al.: The natural history of asymptomatic rotator cuff tears: A threeyear follow-up of fifty cases. J. Bone. Joint. Surg. Am., 95 (14): 1249-55, 2013.

14- TASHJIAN R.Z.: Epidemiology, natural history, and indications for treatment of rotator cuff tears. Clin. Sports. Med., 31 (4): 589- 604, 2012.
15- MELLADO J.M., CALMET J., OLONA M., et al.: Surgically repaired massive rotator cuff tears: MRI of tendon integrity, muscle fatty degeneration, and muscle atrophy correlated with intraoperative and clinical findings. AJR. Am. J. Roentgenol., 184 (5): 1456-63, 2005.

16- GOUTALLIER D., POSTEL J.M., GLEYZE P., et al.: Influence of cuff muscle fatty degeneration on anatomic and functional outcomes after simple suture of fullthickness tears. J. Shoulder Elb. Surg., 12 (6): 550-4, 2003.

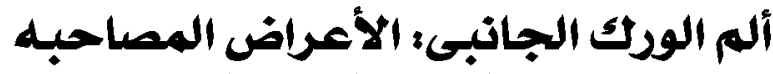

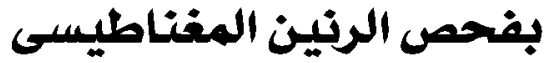

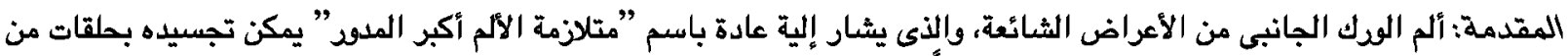

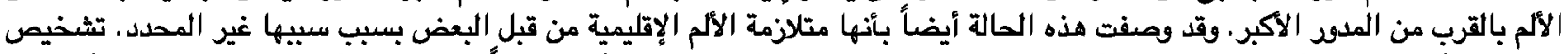

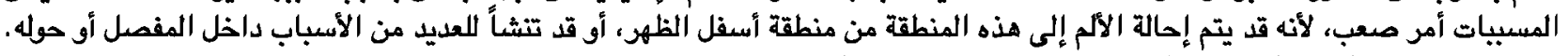

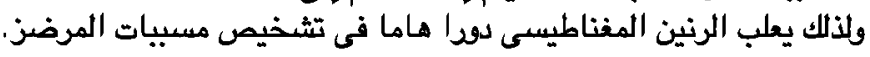

الهدف من البحث: للتعرف على دود التصوير بالرنين المغناطيسى في تقييم آلام الودك الجانبية ودقة في تثخيص الأمراض المسببه.

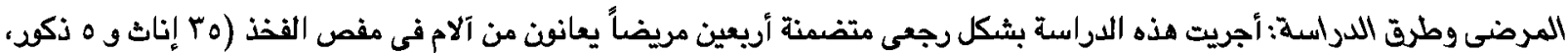

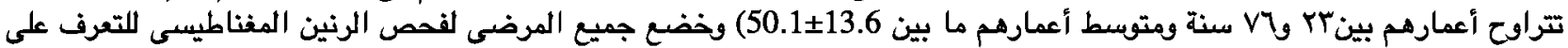

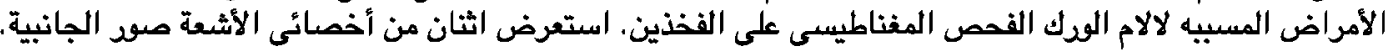

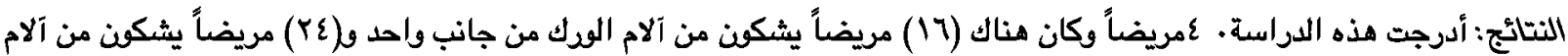

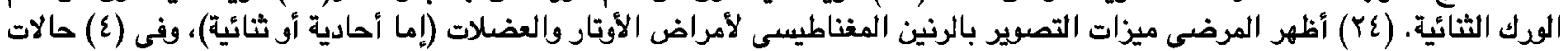

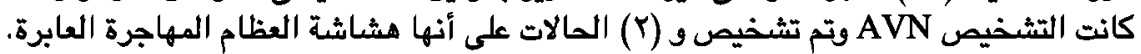

الاستستاجات: يتمتع التصوير بالرنين المغناطيسى بقدرة جيدة لتشخيص الأسباب المختلفة المسبيه لآلام الورك الجانبية وأمراض الأوتار

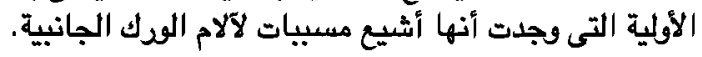

